\title{
Identification of Herpes Simplex Virus Genital Infection: Comparison of a Multiplex PCR Assay and Traditional Viral Isolation Techniques
}

\author{
D.S. Marshall, M.D., D.R. Linfert, B.S., A. Draghi, B.S., Y.S. McCarter, Ph.D., G.J. Tsongalis, Ph.D. \\ Department of Pathology and Laboratory Medicine, Hartford Hospital, Hartford, Connecticut
}

Genital herpes simplex virus (HSV) is of major public health importance, as indicated by the marked increase in the prevalence of genital herpes over the past two decades. Viral culture has traditionally been regarded as the gold standard for diagnosis. In this study, we compared viral culture and the amplification of HSV DNA by the polymerase chain reaction (PCR) with respect to sensitivity, cost, clinical utility, and turnaround time. Patient sample swabs from 100 individuals were inoculated onto MRC-5 cells for isolation. Positive results were confirmed via a direct fluorescent antibody technique, and serotyping, when requested, was performed using HSV-1 and -2-type-specific sera. PCR techniques employed an extraction step of the same initial swab specimen, followed by PCR amplification, using a multiplex assay for HSV-1, 2 DNA. HSV-positive results were found in $32 / 100$ samples via culture and in 36/100 samples via PCR. PCRpositive results yielded $16(44 \%)$ patients infected with HSV-1 and $20(56 \%)$ patients infected with HSV-2. Turnaround time for viral culture averaged 108 hours for positive results and 154 hours for negative results; PCR turnaround time averaged 24-48 hours. Laboratory cost using viral culture was $\$ 3.22$ for a negative result and $\$ 6.49$ for a positive result (including direct fluorescent antibody). Serotyping added $\$ 10.88$ to each culture-positive test. Although laboratory costs for PCR were higher at \$8.20/sample, reimbursement levels were also higher. We propose a multiplex PCR assay for diagnosis of HSV-1 and HSV-2 from patient swabs for use in a routine clinical laboratory setting. This assay offers increased sensitivity, typing, and im-

Copyright () 2001 by The United States and Canadian Academy of Pathology, Inc.

VOL. 14, NO. 3, P. 152, 2001 Printed in the U.S.A

Date of acceptance: December 11, 2000.

The data presented in this manuscript were presented as a platform presentation by D.S.M. at the International Academy of Pathology meeting, New Orleans, LA, March 28, 2000.

Address reprint requests to: G.J. Tsongalis, Department of Pathology and Laboratory Medicine, Hartford Hospital, 80 Seymour Street, Hartford, CT 06102; e-mail: gtsonga@harthosp.org; fax: 860-545-5206. proved turnaround time when compared with traditional viral culture techniques. Although it appears that PCR testing in a routine clinical laboratory setting is cost prohibitive compared with the case of nonserotyped viral culture, it may be very useful when clinical utility warrants distinguishing between HSV 1 and 2 and may be cost effective when reimbursement issues are examined.

KEY WORDS: Herpesvirus, Polymerase chain reaction, Diagnostic testing.

Mod Pathol 2001;14(3):152-156

Genital herpes simplex virus (HSV) infection is of major public health importance, having markedly increased in prevalence throughout the past two decades (1-7). Genital HSV is the most common infective cause of genital ulcerative disease in developed countries and is second in prevalence of viral sexually transmitted diseases only to human papilloma virus, which induces anogenital warts, as the most common viral sexually transmitted disease (1).

HSV type 2 and, less commonly, type 1, are the major causes of genital herpes $(1,3)$. HSV genital infection typically manifests itself as crops of painful, disfiguring lesions that chronically recur. As a result, patients frequently suffer significant psychological and psychosexual stigmatization. Subclinical and completely asymptomatic infection are well recognized and appear to play an important role in neonatal herpes infections $(3,4)$. Neonatal herpes infection may have devastating consequences, ranging from limited skin or eye infections to disseminated multiorgan and central nervous system involvement $(5,6)$.

Recently, HSV infection has been implicated as an important cofactor in the acquisition, transmission, and possibly even the progression of the human immunodeficiency virus infection $(2,7,8)$. HSV is also a well-known etiologic agent in cases of encephalitis, aseptic meningitis, and atypical pneumonias, as well as in a variety of other infections. 
Laboratory diagnosis of genital herpes has traditionally relied upon HSV cell culture as its gold standard (9-11). HSV cell culture techniques are quite sensitive and have proven particularly reliable in early-stage genital infections $(12,13)$. Direct antigen enzyme immunoassay (EIA) and immunofluorescent techniques have also been employed in both the identification and serotyping of HSV infections (14-16). EIA techniques have a significantly reduced turnaround time with respect to cell culture: approximately 6 hours, versus $2-7$ days. However, EIA has been shown to be a much less sensitive diagnostic tool when compared with the case of traditional cell culture (14). Both techniques have been proven to be less sensitive as herpes lesions progress to crusting, healing, and reactivation.

Recent advances in the field of molecular pathology allow detection of HSV DNA using the polymerase chain reaction (PCR). Highly sensitive PCR techniques have already proven to be effective in diagnosing similar serotype HSV infections of the central nervous system $(17,18)$. PCR techniques have been shown to be more sensitive than both cell culture and EIA $(11,19)$. PCR assays can also simultaneously detect and type HSV infections. Serotyping of HSV isolates from traditional viral culture requires additional serologic testing.

The aim of this study is to investigate HSV PCR as a diagnostic method for routine use in a clinical laboratory setting. Laboratory cost will also be assessed and compared with that of viral isolation.

\section{MATERIALS AND METHODS}

\section{Clinical Specimens}

Patient samples were collected on Dacron swabs and transported in viral transport media, (BBL viral culturettes; Becton-Dickinson, Cockeysville, MD). Samples from genital sites of 62 females and 38 males with specific request for HSV isolation were tested.

\section{Viral Culture}

Swabs were inoculated onto a single MRC- 5 cell culture tube. Cell cultures were examined daily, Monday through Friday, for 5 days. MRC- 5 cell culture tubes with viral cytopathic effect typical for HSV infection were confirmed using Bartel's bivalent culture reagent (Bartels Inc., Issaquah, WA), employing a direct-fluorescent antibody technique. Confirmed HSV infections were serotyped only at the specific request of the ordering physician. Serotype testing was performed using a directfluorescent antibody technique with HSV-1- and HSV-2-type-specific sera (Syva Microtrak, Behring Diagnostics, Cupertino, CA).

\section{Molecular Analysis}

The same patient sample swabs used for viral isolation were subsequently returned to the remaining transport media and transported to the molecular pathology laboratory. Excess fluid from the swabs was removed by compression against the side of the transport tube. DNA was extracted using the Puregene extraction kit (Gentra Systems, Inc., Minneapolis, MN): briefly, swabs were submerged in cell lysis solution and the solution compressed out of the swabs. Cells and debris were collected by centrifugation. The remaining pellet was then resuspended in cell lysis solution. Subsequent to protein precipitation, the supernatant was removed to a clean microcentrifuge tube containing $300 \mu \mathrm{L}$ of $100 \%$ isopropanol to precipitate DNA. DNA hydration solution was then added and the sample allowed to rehydrate overnight at room temperature or at $65^{\circ} \mathrm{C}$ for 1 hour.

Analysis with primers targeting the TK3 gene (HSV-1) and the POL gene (HSV-2) were performed using a multiplex PCR assay $(20,21)$. Genomic DNA was incubated in a total reaction volume of $50 \mu \mathrm{L}$ containing approximately $40 \mathrm{ng}$ of both forward and reverse gene specific primers (MWG Biotech, Inc., High Point, NC), 2.0 units AmpliTaq Gold (Perkin Elmer, Foster City, CA), $200 \mu$ m each dNTP, 1.5 $\mathrm{mm} \mathrm{MgCl}_{2}, 10 \mathrm{~mm}$ Tris-HCl (pH 8.3), $50 \mathrm{~mm} \mathrm{KCl,}$ and $0.001 \%$ gelatin (Perkin Elmer). Primer sequences were as follows: TK3 forward primer sequence: 5'-AGC GTC TTG TCA TTG GCG AA-3', TK3 reverse primer sequence: 5'-TTT TCT GCT CCA GGC GGA CT-3', POL forward primer: 5'-GTC CCA CCT CAG CGA TCT GCC T-3', and POL reverse primer: 5'-CAG CAG CGA GTC CTG CAC ACA A-3'. DNA was denatured at $94^{\circ} \mathrm{C}$ for 12 minutes before amplification. PCR was accomplished using $30 \mathrm{cy}-$ cles consisting of 30 seconds of denaturation at $94^{\circ} \mathrm{C}, 30$ seconds of annealing at $55^{\circ} \mathrm{C}$, and $30 \mathrm{sec}-$ onds of extension at $72^{\circ} \mathrm{C}$. The final cycle included a 3-minute extension step at $72^{\circ} \mathrm{C}$.

Fragment size analysis was performed by direct visualization of PCR products, 342-bp and 490-bp fragments for TK3 and POL, after gel electrophoresis in a $10 \%$ polyacrylamide gel and staining with ethidium bromide. Control PCR reactions included the following: blank (no target DNA), negative (normal human DNA), and positive (HSV-1 \#08-705000, HSV-2 \#08-706-000; ABI, Columbia, MD) controls for each run.

Molecular analysis was preformed three times each week: Monday, Wednesday, and Friday. All calculations in terms of cost and turnaround time were based on batching 10 samples per PCR run.

\section{RESULTS}

HSV positive results were found in 32 of 100 clinical samples via viral culture and in 36 of 100 
samples via PCR. Four discordant cases were identified (positive via PCR, negative via culture). PCRpositive results yielded $16(44 \%)$ patients infected with HSV-1 and 20 patients infected with HSV-2 (Fig. 1).

With viral culture techniques, turnaround time averaged 108 hours for a positive result and 154 hours for a negative result. Turnaround time using PCR was determined to average 48 hours, with a range from 24 to 72 hours. Although PCR test results could be reported in one day, it was more typical to have a 48-hour turnaround time.

Cost analysis for viral culture techniques show laboratory cost for negative samples to be $\$ 3.22$ and $\$ 6.49$ for positive isolates (includes added cost of confirmatory direct fluorescent antibody testing). Serotyping of viral isolates adds an additional $\$ 10.88$ to each positive isolation result. Laboratory cost using the PCR assay totals $\$ 8.20$ per sample. The cost of testing the 100 clinical samples in this study was $\$ 426.64$ by viral isolation. This cost increased to $\$ 753.04$ if serotyping was added. The total cost of analyzing these same 100 specimens by PCR was $\$ 820.00$ and included typing within the same assay. The recent Medicare reimbursement level for herpes culture was $\$ 36.02$, $\$ 16.58$ for direct fluorescent antibody, and $\$ 27.91$ for serotyping. A single HSV-amplified probe was reimbursed at $\$ 48.50$.

\section{DISCUSSION}

In this study, we have evaluated a multiplex PCR assay to simultaneously identify and type HSV from direct clinical samples. No modifications or changes in routine practice were necessary on the

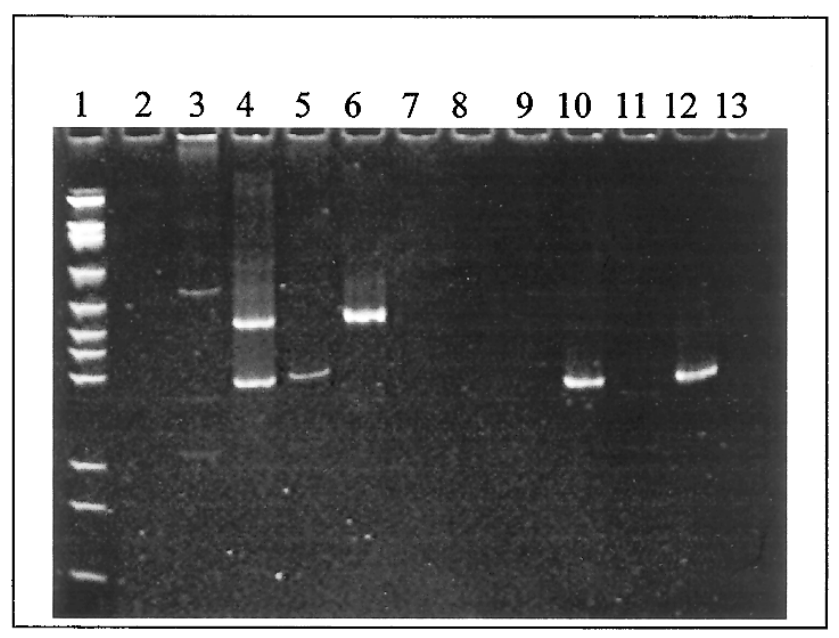

FIGURE 1. Representative HSV 1 and 2 multiplex PCR gel. Molecular size markers, Lane 1; blank control, Lane 2; negative control, Lane 3; positive control, Lane 4; positive result for HSV-1, Lanes 5, 10, 12; positive result for HSV-2, Lane 6. Lanes 5-13 represent HSV typing from submitted clinical specimens. part of the clinicians. In fact, the same sample used for viral culture isolation was later used for our PCR assay.

The sensitivity of our PCR assay was similar to that in previous investigations that have demonstrated the greater sensitivity of PCR when compared with viral isolation techniques $(9,11)$. Factors implicated in the reduced sensitivity for culture are manifold. Increased transit time between specimen collection and inoculation into cell culture seems to considerably reduce the infectivity that can occur during prolonged transport $(12,13)$. Additionally, recurrent HSV infections, as well as lesions that are in healing stages, are much less likely to yield positive viral cultures for $\operatorname{HSV}(1,6)$. On the other hand, like herpes-specific culture, PCR analysis detects only the target virus but does not yield an isolate for further testing. For general virus detection, extended multiplex PCR analysis or microarray technology would be warranted. Advances in in vitro amplification technologies, including automated platforms, will make these types of molecular technologies more suitable for the clinical laboratory that does not have the currently accepted facilities.

In the present study, we identified four discordant cases in which PCR was positive and viral isolation techniques negative. The medical history of these individuals was significant for longstanding genital herpes infection in three of the four patients in question. The fourth patient had no known history of HSV infection but did have genital lesions that were clinically suspicious for herpes. All four patients were counseled and treated for genital herpes infection.

Although PCR is known to be more sensitive than culture, it also allows for simultaneous typing of HSV 1 and 2 infections. HSV serotyping is not routinely performed by all viral isolation laboratories and typically requires additional testing that adds significantly to laboratory cost and turnaround time.

Traditionally, HSV-2 has been associated with most genital infections, with HSV-1 assuming a much less significant role (2). However, studies over the past decade have demonstrated the increasing prevalence of HSV-1 in genital infections. Several studies have reported the prevalence of HSV-1 at $20-60 \%$ (2). Some authors have suggested that HSV-1 genital infection may be a consequence of oral-genital contact (20). In contrast, HSV-2 infections are spread via genital-genital contact and are frequently associated with multiple sexual partners (4). HSV-1 genital infections may have a lower rate of clinical recurrence when compared with HSV-2 infections. Thus, in addition to its importance from an epidemiologic standpoint, viral typing may provide information useful for prognostication of the natural history of a particular infection (22). This 
information may be of potential value to clinicians in the counseling of their patients.

In adopting a new test for use in the clinical laboratory, turnaround time and cost are important factors to consider. Turnaround time for viral culture can depend on many factors, which includes a variety of individual laboratory policies and techniques. Turnaround time was measured as the time a clinical specimen was logged in as received in the lab until a diagnostic result was recorded. With viral isolation, turnaround time averaged 108 hours for a positive result and 154 hours for a negative result. Turnaround time as well as all cost calculations for PCR were based on batching of clinical samples, with PCR performed three times each week (Monday, Wednesday, Friday). Turnaround time for PCR ranged from 24 to 72 hours, with an average estimated turnaround time of 48 hours. Improved turnaround time, coupled with increased sensitivity, gives PCR a distinct advantage over isolation techniques as a diagnostic tool.

A laboratory cost analysis determined the cost of this PCR assay to be $\$ 8.20$ per clinical sample tested. This is based on batching 10 clinical samples per run. Cost for viral culture techniques will vary depending on whether culture has yielded a positive or negative result. A negative result in our laboratory costs $\$ 3.22$ and a positive result $\$ 6.49$ because of the added cost of a direct-fluorescent confirmatory test. Recall however that these results provide no information as to HSV type, which, when performed in our laboratory, adds an additional $\$ 10.88$ to the cost of a positive test result.

When extrapolated to the patient samples from our study, the total cost for PCR was $\$ 820$. Total cost for viral isolation was $\$ 426.64$ (without additional serotype testing on positive samples). Cost for viral isolation including serotyping would have totaled $\$ 753.04$ if performed on each positive case. This cost is relatively comparable to the $\$ 820$ total cost for PCR. Although reimbursement levels vary geographically and by payor, it appears from recent data we obtained that reimbursement levels may be higher for molecular testing. In addition, one needs to consider labor cost savings. However, despite the potential cost benefits, this does raise the question regarding the necessity of typing HSV genital specimens. In our 100 patient samples, none of the clinicians requested typing. In fact, typing is only requested approximately two to three times each year in our laboratory. It is assumed that although HSV typing of genital infections may be important in terms of epidemiology and academic interest, at the present time, it offers little clinical utility to physicians in the management of infected patients.

In summary, we have established a multiplex PCR assay for the identification and typing of HSV genital infections. We propose that PCR used in a routine clinical laboratory setting offers increased sensitivity, simultaneous typing, and improved turnaround time when compared with traditional viral isolation techniques. Although one can debate reimbursement and cost savings issues, we feel that workflow, clinical utility, and service should also be of significant consideration when making such technological decisions.

Acknowledgments: The authors thank Rocky Ackroyd, Kelly Ennis, and Irene Ratkiewicz for technical assistance in completion of this project.

\section{REFERENCES}

1. Kinghorn GR. Genital herpes: natural history and treatment of acute episodes. J Med Virol 1993;(1 Suppl):33-8.

2. Kinghorn GR. Epidemiology of genital herpes. J Int Med Res 1994;22(1 Suppl):14A-23A.

3. Mertz GJ. Epidemiology of genital herpes infections. Infect Dis Clin North Am 1993;7:825-39.

4. Slomka MJ. Seroepidemiology and control of genital herpes. The value of type-specific antibodies to herpes simplex virus. Commun Dis Rep Rev 1996;6:R41-5.

5. Corey L. Genital herpes. In: Holmes K, Mardh PA, Sparling FP, Wiesner PJ editors. Sexually transmitted diseases. New York: McGraw Hill Information Services Company; 1990.

6. Corey L. The current trend in genital herpes: progress in prevention. Sex Transm Dis 1994;21(2 Suppl):S38-44.

7. Stamm WE, Handsfield HH, Rompalo AM, Ashley RL, Roberts PL, Corey L. The association between genital ulcer disease and acquisition of HIV infection in homosexual men. JAMA 1988;260:1429-33.

8. Hook EW, Cannon RO, Nahmias AJ, Lee FF, Campbell CH Jr, Glasser D, et al. Herpes simplex virus infection as a risk factor for human immunodeficiency virus infection in heterosexuals. J Infect Dis 1992;165:251-5.

9. Slomka MJ, Emery L, Munday PE, Moulsdale M, Brown DWG. A comparison of PCR with virus isolation and direct antigen detection for diagnosis and typing of genital herpes. J Med Virol 1998;55:177-83.

10. Ashley RL. Laboratory techniques in the diagnosis of herpes simplex infection. Genitourin Med 1993;69:174-83.

11. Cone RW, Hobson AC, Brown Z, Ashley R, Berry S, Winter C, et al. Frequent detection of herpes simplex virus infection by polymerase chain reaction among pregnant females. JAMA 1994;272:792-6.

12. Jensen C, Johnson FB. Comparison of various transport media for viability maintenance of herpes simplex virus, respiratory syncytial virus, and adenovirus. Diagn Microbiol Infect Dis 1994;19:137-42.

13. Skinner GRB, Billstrom MA, Randall S, Ahmad A, Patel S, Davies J, et al. A system for isolation, transport, and storage of herpes simplex viruses. J Virol Meth 1997;65:1-8.

14. Verano L, Michalski FJ. Comparison of a direct antigen enzyme immunoassay, Herpchek with cell culture for detection of herpes simplex virus from clinical specimens. J Clin Microbiol 1995;33:1378-9.

15. Scicchitano LM, Shetterly B, Boubeau PP. Evaluation of light diagnostics SimulFluor HSV/VZV immunofluorescence assay. Diagn Microbiol Infect Dis 1999;35:205-8.

16. Coyle PV, Desai A, Wyatt D, McCaughey C, O'Neill HJ. A comparison of virus isolation, indirect immunofluorescence and nested multiplex polymerase chain reaction for the di- 
agnosis of primary and recurrent herpes simplex type 1 and 2 infections. J Virol Methods 1999;83:75-82.

17. Cassinotti P, Mietz H, Siegl G. Suitability and clinical application of a multiplex nested PCR assay for the diagnosis of herpes simplex infections. J Med Virol 1996;50:75-81.

18. Lakeman FD, Whitley RJ, National Institute of Allergy and Infectious Diseases Collaborative Antiviral Study Group. Diagnosis of herpes simplex virus encephalitis: application of polymerase chain reaction to cerebrospinal fluid from brainbiopsied patients and correlation with disease. J Infect Dis 1995;171:857-63.

19. Diaz-Mitoma F, Ruben M, Sacks S, MacPherson P, Caissie G. Detection of viral DNA to evaluate outcome of antiviral treatment of patients with recurrent herpes genital herpes. J Clin Microbiol 1996;34:657-63.
20. Bai X, Hosler G, Rogers BB, Dawson DB, Scheuermann RH. Quantitative polymerase chain reaction for human herpesvirus diagnosis and measurement of Epstein-Barr virus burden in posttransplant lymphoproliferative disorder. Clin Chem 1997;43:1843-9.

21. Hoang MP, Rogers BB, Dawson DB, Scheuerman RH. Quantitation of 8 human herpesviruses in peripheral blood of human immunodeficiency virus-infected patients and healthy blood donors by polymerase chain reaction. Am J Clin Pathol 1999;111:655-9.

22. Lafferty WE, Coombs RW, Bedetti J, Critchlow C, Corey L. Recurrences after oral and genital herpes simplex virus infection. Influence of site of infection and viral type N Engl J Med 1987;316:1444-9.

\section{Book Review}

\section{Emory TS, Carpenter HA, Gostout CJ, Sobin LH: Atlas of Gastrointestinal Endoscopy \& Endoscopic Biopsies, 448 pp, Washington, DC, Armed Forces Institute of Pathology, 1999 (\$90.00).}

Like many other hospital pathologists, I got used to receiving biopsies without too many clinical data. And then one day last year our gastroenterologists started sending us not only copies of their endoscopic findings but also photographs of what they saw at the other end of the tube. In the meanwhile, having personally seen how such an 'improved communication' between clinicians and pathologists had benefited several patients, I became convinced that correlating 'their' and 'our' pictures was not only the best way of dealing with intestinal lesions, but the only way that I would recommend to anybody practicing this diagnostic art.

The present book is a wonderful example how the virtual 'divide' between the endoscopists and pathologists could and should be bridged. Based on the material culled from the files of the Mayo Clinic and the Armed Forces Institute of Pathology, it is a comprehensive primer for both pathologists and gastroenterologists entering the field. It covers all the major entities, illustrating them with high-quality photographs taken through the endoscope or the microscope. The pictures are most informative, well-chosen, and of high quality. The text that accompanies the pictures is succinct and to the point, complementing and enhancing the educational value of the pictorial material.

Instead of criticism, I am including a few suggestions for revision in the second edition, which I am sure will be coming out in not so distant future. To improve the didactic value of the pictures, I would annotate them or insert markings. This would help pathologists understand better the endoscopic photographs, and I am sure that the clinicians would not object to some assistance in interpreting the microphotographs. Furthermore, I would replace some of the endoscopic photos that show too much reflected light. Some entities have only endoscopic pictures and should be illustrated with equivalent microphotographs. The index also could be a bit more detailed and user friendly, and could include some alternative names if not even some 'jargon terms' from both sides of the clinicopathologic divide.

By all accounts this is a well-done (pun not intended!) G.I. book. At the current price it is a bargain that should not be bypassed by pathologists or gastroenterologists. It should serve them well on their tortuous route through the alimentary tract.

\section{Ivan Damjanov \\ University of Kansas School of Medicine Kansas City, Kansas}

